\title{
A Comparative Study of TAVR versus SAVR in Moderate and High-Risk Surgical Patients: Hospital Outcome and Midterm Results
}

\author{
Ahmed Abdelgawad, MD, ${ }^{1}$ Mohamed A. Hussein, MD,${ }^{1}$ Hesham Naeem, MD, ${ }^{2}$ Reda Abuellata, MD, ${ }^{2}$ \\ Salah Alghamdy, SBC, ${ }^{2}$ \\ ${ }^{1}$ Departments of Cardiac Surgery and ${ }^{2}$ Cardiology, Madinah Cardiac Centre, Kingdom of Saudi Arabia
}

\section{ABSTRACT}

Background: Although the use of transcatheter aortic valve replacement (TAVR) has recently become an attractive strategy in prohibitive surgical high-risk patients undergoing aortic valve replacement (AVR), the most appropriate treatment option in patients with an intermediate- to high-risk profile- whether conventional surgery (SAVR) or TAVR — has been widely debated.

Methods: One hundred and forty-three consecutive patients with intermediate to high risk were prospectively enrolled and selected to undergo SAVR (Group 1 [G1], $\mathrm{n}=63$ ) or TAVR (Group 2 [G2], $\mathrm{n}=80$ ) following a multidisciplinary evaluation including frailty, anatomy, and degree of atherosclerotic disease of the aorta/peripheral vessels. The mean logistic EuroSCORE $(\mathrm{G} 1=20.11 \pm 7.144$ versus $\mathrm{G} 2=23.33 \pm 8.97 ; P=.022), \mathrm{STS}$ score $(\mathrm{G} 1=5.722 \pm 1.309$ versus $\mathrm{G} 2=5.958 \pm 1.689 ; P=.347)$, and preoperative demographics such as sex, left ventricular ejection fraction (LVEF), body mass index (BMI), peripheral vascular disease, diabetes, atrial fibrillation, renal impairment and syncope were similar. Of note, chronic obstructive pulmonary disease was more frequent in TAVR patients (G2 [46.2\%] versus $\mathrm{G} 1[19.0 \%] ; P=.001)$, whereas pulmonary hypertension was more frequent in SAVR group (G1 [47.6\%] versus G2 [17.5\%]; $P=.000)$. The SAVR was performed with either a mechanical or tissue valve; meanwhile, TAVR was performed with either Core valve prosthesis or Edwards-Sapiens XT valve.

Results: SAVR group showed higher incidence of some postoperative complications compared to TAVR, namely, postoperative bleeding ( $4.8 \%$ versus $0.0 \% ; P=.048)$, tamponade $(4.8 \%$ versus $0.0 \% ; P=.048)$ and postoperative atrial fibrillation $(34.9 \%$ versus $10.0 \% ; P=.000)$, whereas TAVR group had a higher incidence of other sets of postoperative complications, namely, left bundle branch block $(58.8 \%$ versus $4.8 \% ; P=.000)$, need for permanent pacemaker implantation $(25.0 \%$ versus $1.6 \% ; P=.000)$ and peripheral vascular complications ( $15.0 \%$ versus $0.0 \% ; P=.001)$. On the contrary, when the two groups were compared they did not show any significant difference regarding anemia requiring more than two units of blood transfusion, postoperative renal failure, stroke, myocardial infarction, and hospital mortality.

Received October 6, 2018; accepted December 12, 2018.

Correspondence: Abmed Abdelgawad, MD, Associate Consultant Cardiac Surgeon, Madinab Cardiac Centre; +966-549-567-842 (e-mail: a_ewais@yahoo.com).
$P=.534, .873, .258, .373$ and .072 respectively. Hospital mortality was similar among the two groups $(\mathrm{G} 1=0 \%$ versus $\mathrm{G} 2$ $=5 \% ; P=.072)$. At the 24-month follow-up, overall mortality, major adverse cardiac and cerebrovascular events were comparable between the two groups but prosthetic regurgitation was better in SAVR group $(\mathrm{G} 2=8$ patients $[10.0 \%]$ versus $\mathrm{G} 1$ $=1$ patient $[1.6 \%]$ in SAVR group; $P=.040$ ).

Conclusion: In this study, we could not detect an advantage in survival when SAVR or TAVR were utilized in intermediate to high surgical risk patients needing aortic valve replacement for severe aortic stenosis.

\section{INTRODUCTION}

Left untreated, severe symptomatic aortic stenosis has had a dismal outcome with as high as $30-50 \%$ one-year mortality [Ben-Dor 2010; Turina 1987; Leon 2010]. Since the introduction of TAVI in 2002 [Cribier 2002], technology has played its role to reach a stage where this modality imposes itself in the armamentarium of clinical surgical practice with high expectations and very good outcomes.

Even though surgical aortic valve replacement (AVR) still represents the gold standard among the therapeutical options in patients with severe aortic valve stenosis [Ben-Dor 2010], the use of TAVI became the standard of care (Class 1) indication to treat prohibitive high-risk surgical patients with acceptable outcomes [Nishimura 2017]. Moreover, a wider scale of use of TAVI in patients with intermediate to high risk has also been introduced, although the early and midterm outcomes of a conventional surgical versus TAVI in this specific situation have not been investigated enough Cribier 2002, Nishimura 2017]. However, recent guidelines refer to the superiority of SAVR in the wake of available data in this matter. Therefore, we sought to investigate the clinical outcomes of patients with severe aortic valve stenosis and an intermediate- to high surgical risk profile following conventional surgical (SAVR) versus transcatheter aortic valve replacement (TAVR).

\section{METHODS}

\section{Study Population}

From October 2010 to February 2013, after Institutional Review Board approval, we started a retrospective cohort 
Table 1. Patients' Demographics

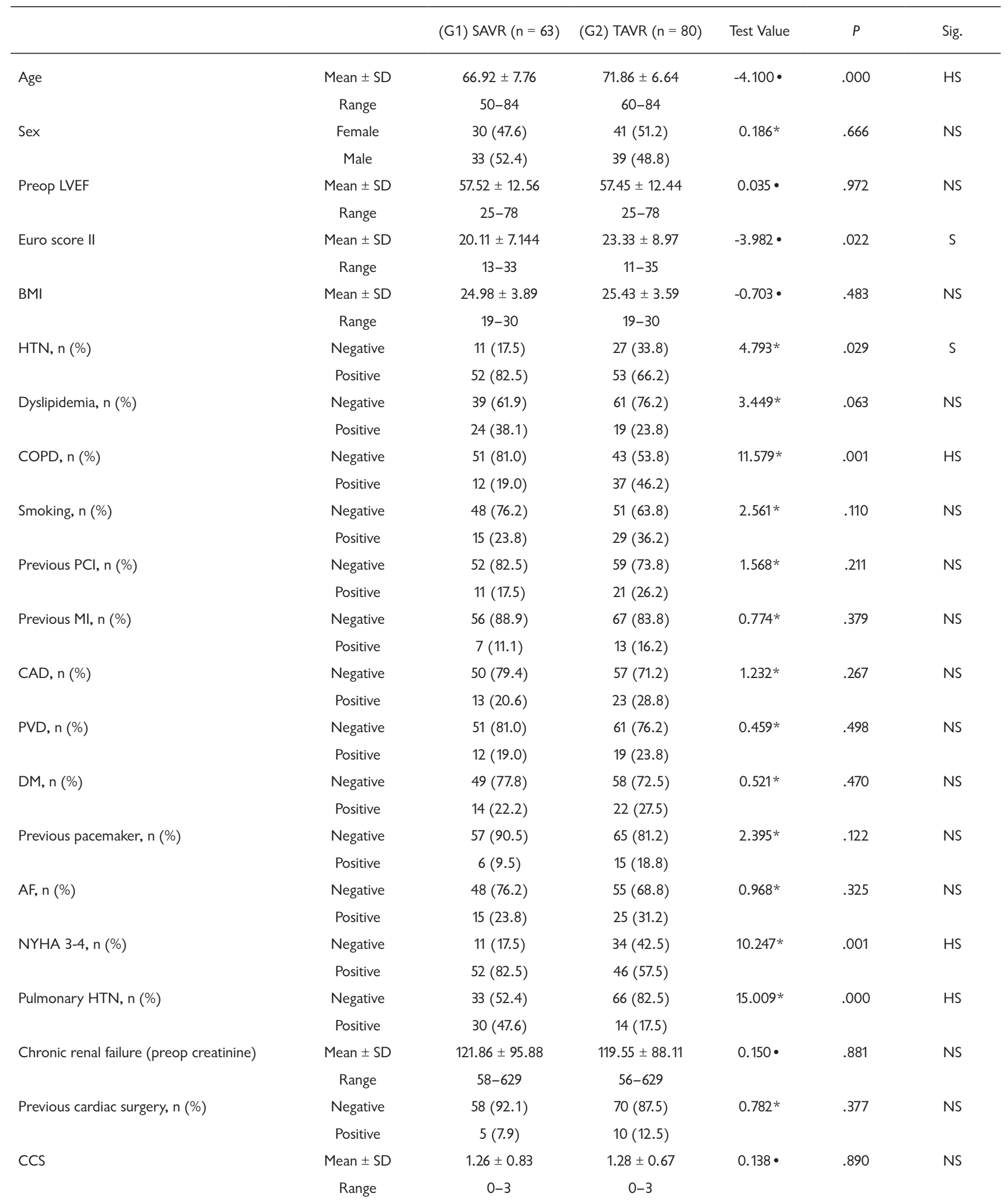


Table 1. Patients' Demographics [Cont.]

\begin{tabular}{lccccc}
\hline & $(\mathrm{G} 1) \operatorname{SAVR}(\mathrm{n}=63)$ & $(\mathrm{G} 2)$ TAVR $(\mathrm{n}=80)$ & Test Value & $P$ & Sig. \\
\hline Syncope & Mean \pm SD & $1.1 \pm 0.6$ & $1.2 \pm 0.7$ & $-0.817 \cdot$ & .416 \\
STS Score & Range & $0-2$ & $0-2$ & .347 \\
\hline
\end{tabular}

G1 indicates group 1; G2, group 2; SAVR, surgical aortic valve replacement; TAVR, transcatheter aortic valve replacement; preop LVEF, preoperative left ventricular ejection fraction; BMI, body mass index; HTN, hypertension; COPD, chronic obstructive pulmonary disease; previous $\mathrm{PCl}$, previous percutaneous coronary intervention; previous MI, previous myocardial infarction; CAD, coronary artery disease; PVD, peripheral vascular disease; DM, diabetes mellitus; AF, atrial fibrillation; NYHA, New York Heart Association functional class; pulmonary HTN, pulmonary hypertension; CCS, Canadian Cardiovascular Society grading of angina pectoris; NS, not significant; S, significant; HS, highly significant.

$\bullet$, Independent t-test; *, Chi-square test.

study: 143 consecutive patients with severe aortic valve stenosis and an intermediate- to high-risk profile were retrospectively enrolled and underwent standard aortic valve replacement (SAVR, Group 1 [G1], n = 63), or TAVR (Group 2 [G2], $\mathrm{n}=80$ ). The decision of which procedure should be performed in each case was taken after a multidisciplinary evaluation by the Heart Team (composed of cardiac surgeons, interventional cardiologists and a cardiac anesthesiologist), considering frailty, anatomy, and degree of atherosclerotic disease of the aorta and peripheral vessels. The inclusion criteria were: 1. Patient had severe aortic valve stenosis with echocardiographically derived criteria: mean gradient $>40 \mathrm{mmHg}$ or jet velocity greater than $4.0 \mathrm{~m} / \mathrm{s}$ and an initial aortic valve area (AVA) of $<0.8 \mathrm{~cm}^{2}$ (or AVA index $<0.5 \mathrm{~cm}^{2} / \mathrm{m}^{2}$ ). 2. Patient was symptomatic from his/her aortic valve stenosis, as demonstrated by NYHA Functional Class II or greater. 3. The heart team agreed on eligibility including assessment that TAVR or SAVR is appropriate for the patient. 4. STS score $>4$. Exclusion criteria were: 1 . Evidence of an acute myocardial infarction $\leq 1$ month (30 days); before the intended treatment [defined as: Q wave MI, or non $\sim$ wave $\mathrm{MI}$ with total CK elevation of $\mathrm{CK} \sim \mathrm{MB} \geq$ twice normal in the presence of $M B$ elevation and/or troponin level elevation (WHO definition). 2. Mixed aortic valve disease (aortic stenosis and aortic regurgitation with predominant aortic regurgitation $>3+$ ). 3 . Preexisting mechanical or bioprosthetic valve. 4. Complex coronary artery disease: (a) Unprotected left main coronary artery (b) Syntax score $>32$ (in the absence of prior revascularization). 5. Hypertrophic cardiomyopathy with or without obstruction (HOCM). 6. Severe ventricular dysfunction with LVEF $<20 \%$. 7. Echocardiographic evidence of intracardiac mass, thrombus or vegetation. 8. Active upper GI bleeding within 3 months (90 days) prior to procedure. 9. Clinically (by neurologist) or neuroimaging confirmed stroke or transient ischemic attack (TIA) within 6 months (180 days) of the procedure. 10. Active bacterial endocarditis within 6 months (180 days) of procedure.

\section{Transfemoral Aortic Valve Replacement Group}

We reviewed the data of 80 patients who underwent transfemoral aortic valve implantation from October 2010 to
February 2013 at Madinah Cardiac Center, Kingdom of Saudi Arabia. The transfemoral route was utilized in all cases of the current study population. The main indication for TAVI was severe aortic valve stenosis (aortic valve area, $<0.8 \mathrm{~cm}^{2}$; mean transaortic gradient, $>40 \mathrm{mmHg}$ associated with 1 or more of the following: (1) porcelain aorta; (2) high surgical risk [logistic Euroscore, $>20 \%$ ] and (3) other serious comorbidities, including severe pulmonary disease, previous chest irradiation, or severe liver disease. TAVI procedures were usually performed and the only implanted devices were the SAPIEN or SAPIEN XT pericardial balloon expandable bioprosthesis (Edwards LifeSciences, Irvine, CA). The absolute contraindications for TAVI was extremely poor left ventricular ejection fraction $(<15 \%)$.

\section{Surgical Aortic Valve Replacement Group}

We retrospectively collected data of 63 consecutive patients who had undergone isolated SAVR from October 2010 to February 2013 at Madinah Cardiac Center, Kingdom of Saudi Arabia. All SAVR procedures were performed through full sternotomy, with moderate hypothermic cardiopulmonary bypass. Cold-blood intermittent cardioplegia was usually administered in both an antegrade and a retrograde fashion. Prostheses were implanted with 2-0 braided pledgeted horizontal mattress sutures (pledgets on the ventricular side). Bioprostheses and mechanical prostheses were used in $52(82.5 \%)$ and $11(17.5 \%)$ patients, respectively. The mean aortic crossclamp and cardiopulmonary bypass time was $47.79 \pm 8.34$ and $80.38 \pm 9.02$ minutes, respectively. The patients in all groups underwent clinical and echocardiographic assessment at the study site before the procedure and at hospital discharge. Echocardiographic measurements were done according to the current recommendations [Lang 2006]. Prosthetic aortic regurgitation (AR) was classified as none or trace, mild $(1+/ 3+)$, moderate $(2+/ 3+)$, or severe $(3+/ 3+)$ according to recent recommendations [Zoghbi 2009]. Study primary endpoints were either short term (such as early postoperative complications and hospital mortality - 30-day mortality) as well as overall survival, survival free from major adverse cardiac and cerebrovascular events (MACCEs), defined as cardiac-related mortality, myocardial infarction, 
Table 2. Intraoperative Data

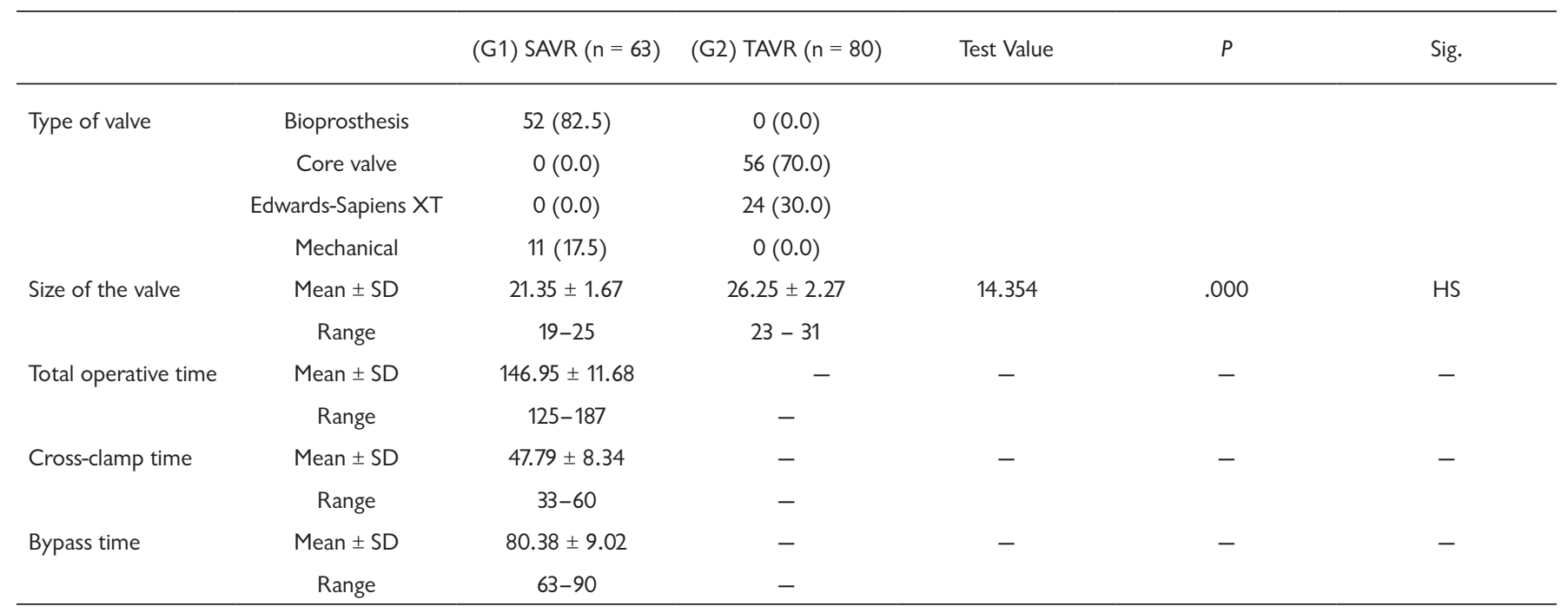

NS indicates not significant; S, significant; HS, highly significant.

cerebrovascular accidents, and major hemorrhagic events as per VARC Guidelines [Kappetein 2012]; and a composite end-point of survival free from MACCEs and prosthesis dysfunction, such as periprosthetic aortic regurgitation $\geq 2$.

\section{Statistical Analysis}

Pre- and postoperative variables were analyzed using the Fisher exact test and the Pearson chi-square test for discrete variables and the Mann-Whitney $U$ test for continuous variables; values for continuous variables are expressed as mean \pm standard deviation. Survival curves were evaluated by means of Kaplan-Meier analysis. A $P$ value $<.05$ was considered to be significant. The statistical package utilized was the SPSS software (Version 19, IBM, New York, NY, USA).

\section{RESULTS}

\section{Population Characteristics}

Preoperative patient characteristics are outlined in Table 1: on the one hand, there was no difference among the groups in terms of sex, left ventricular ejection fraction (LVEF), body mass index (BMI), peripheral vascular disease, diabetes, atrial fibrillation, renal impairment and syncope. P values were .666, $.972, .483, .498, .470, .325, .881$ and .416 respectively. On the other hand, we noticed that the TAVR group were older in age $(71.86 \pm 6.64$ versus $66.92 \pm 7.76 ; P=.000)$, having higher EuroSCORE $(\mathrm{G} 1=20.11 \pm 7.144$ versus $\mathrm{G} 2=23.33 \pm 8.97$; $P=.022)$, higher STS score albeit with no significance (G1 $=5.722 \pm 1.309$ versus $\mathrm{G} 2=5.958 \pm 1.689 ; P=.347)$, and higher incidence of COPD (46.2\% versus $19.0 \% ; P=.001)$. The SAVR group, however, had a more advanced NYHA score $(82.5 \%$ versus $57.5 \% ; P=.001)$, pulmonary hypertension $(47.6 \%$ versus $17.5 \% ; P=.000)$ and were more likely to be hypertensive $(82.5 \%$ versus $66.2 \% ; P=.029)$.

\section{Intraoperative Data}

Transfemoral approach was used universally in our TAVR patients compared to median sternotomy that was used solely for our conventional SAVR. Moreover, 56 patients $(70.0 \%)$ had Core valve versus 24 patients $(30.0 \%)$ who had EdwardsSapiens XT valve in TAVR group. In SAVR group, 52 patients $(82.5 \%)$ had tissue valves implanted versus 11 patients $(17.5 \%)$ who had mechanical valves implanted. As shown in Table 2, the overall size of the valve implanted was significantly higher in TAVR group $(26.25 \pm 2.27$ versus $21.35 \pm 1.67 ; P=.000)$.

\section{Early Postoperative Follow-up}

Table 3 shows the immediate postoperative results of operated patients. SAVR had lengthier mechanical ventilation hours $(9.52 \pm 3.10$ versus $2.00 \pm 1.04 ; P=.000)$, ICU stay in hours $(57.81 \pm 12.11$ versus $27.71 \pm 2.45 ; P=.000)$ and hospital stay in days $(13.63 \pm 13.31$ versus $4.98 \pm 1.42 ; P=.000)$. Moreover, SAVR group showed higher incidence of some postoperative complications compared to TAVR, namely, postoperative bleeding (4.8\% versus $0.0 \% ; P=.048)$, tamponade ( $4.8 \%$ versus $0.0 \%$; $P=.048)$ and postoperative atrial fibrillation $(34.9 \%$ veruss $10.0 \% ; P=.000)$, whereas TAVR group had a higher incidence of other sets of postoperative complications, namely, left bundle branch block (58.8\%) versus $4.8 \% ; P=.000)$, need for permanent pacemaker implantation $(25.0 \%$ versus $1.6 \% ; P=.000)$ and peripheral vascular complications $(15.0 \%$ versus $0.0 \%$; $P=.001)$. On the contrary, when the two groups were compared, they did not show any significant difference in regard to anemia requiring more than two units of blood transfusion, postoperative renal failure, stroke, myocardial infarction, and hospital mortality $(P=.534, .873, .258, .373$ and .072 respectively).

\section{Echocardiograghy and Hemodynamic Performance}

Preoperative and midterm postoperative echo data are illustrated in Table 4. As far as preoperative transthoracic echo 
Table 3. Early Postoperative Data

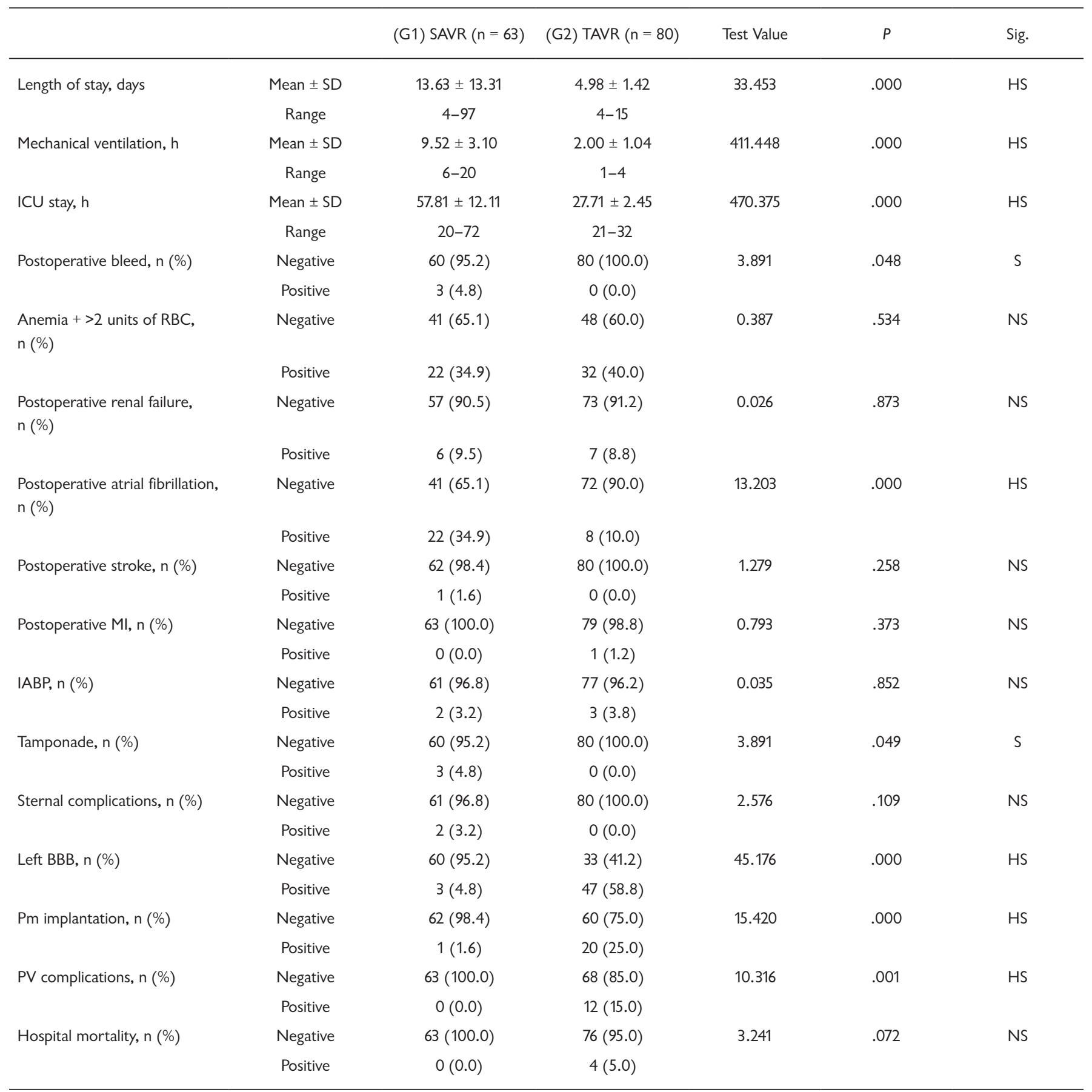

ICU indicates intensive care unit; postoperative MI, postoperative myocardial infarction; IABP, intraaortic balloon counter pulsation; Left BBB, left bundle branch block; Pm implantation, permanent pacemaker implantation; PV complications, peripheral vascular complications; NS, not significant; S, significant; HS, highly significant.

is concerned, baseline size of the aortic annulus in $\mathrm{mm}$ and aortic valve area in $\mathrm{cm}^{2}$ were similar among the two groups $(\mathrm{G} 1=24.97 \pm 5.85 \mathrm{~mm}$ versus $\mathrm{G} 2=25.08 \pm 3.42 \mathrm{~mm} ; P=$ $.892 ;$ and $\mathrm{G} 1=0.80 \pm 0.19$ versus $\mathrm{G} 2=0.82 \pm 0.19 ; P=.707$ respectively). Other preoperative hemodynamic parameters were also similar such as preoperative peak gradient and preoperative mean gradient $(P=.921$ and 0.269 respectively). Two years postoperative transthoracic echo for the two groups 
Table 4. Pre- and Early Postoperative Echo and Hemodynamic Performance

\begin{tabular}{|c|c|c|c|c|c|c|}
\hline \multicolumn{7}{|l|}{ Mean preoperative } \\
\hline Preoperative annulus, mm & Mean \pm SD (range) & $24.97 \pm 5.85(17-39)$ & $25.08 \pm 3.42(17-39)$ & -0.136 & .892 & NS \\
\hline Preoperative valve area, $\mathrm{cm}^{2}$ & Mean \pm SD (range) & $0.80 \pm 0.19(0.5-1.1)$ & $0.82 \pm 0.19(0.5-1.1)$ & -0.377 & .707 & NS \\
\hline Preoperative LVEF & Mean \pm SD (range) & $57.52 \pm 12.56(25-78)$ & $57.45 \pm 12.44(25-78)$ & 0.035 & .972 & NS \\
\hline Postoperative peak gradient, $\mathrm{mmHG}$ & Mean \pm SD (range) & $24.90 \pm 12.75(10-64)$ & $19.70 \pm 6.51(10-49)$ & 3.166 & .002 & $\mathrm{HS}$ \\
\hline Postoperative mean gradient, $\mathrm{mmHG}$ & Mean \pm SD (range) & $15.83 \pm 9.46(6-73)$ & $13.30 \pm 8.58(6-73)$ & 1.670 & .097 & NS \\
\hline \multicolumn{7}{|l|}{$A R>2$} \\
\hline Negative (\%) & & $62(98.4)$ & $72(90.0)$ & 4.230 & .040 & NS \\
\hline
\end{tabular}

Preoperative LVEF indicates preoperative left ventricular ejection fraction; AR, aortic regurgitation; postoperative LVEF, postoperative left ventricular ejection fraction; NS, not significant; S, significant; HS, highly significant.

showed successful decrease of both peak and mean gradient $(\mathrm{G} 1=15.83 \pm 9.46$ versus $\mathrm{G} 2=13.30 \pm 8.58 ; P=.097)$ albeit with higher peak gradient in SAVR group referring to a better hemodynamic performance for TAVR group (G1 24.90 \pm G2 12.75 versus $19.70 \pm 6.51 ; P=.002)$. Furthermore, a degree of at least moderate periprosthetic aortic regurgitation was found mainly in patients undergoing TAVR implantation (8 patients $[10.0 \%]$ versus 1 patient $[1.6 \%]$ in SAVR group; $P=.040)$.

\section{MACCE and Readmission at Midterm Follow-up}

Table 5 demonstrates that overall mortality (cardiac and non-cardiac related) was similar among the groups $(\mathrm{G} 1=3$ patients [4.8\%] versus $\mathrm{G} 2=10$ patients $[12.5 \%] ; P=.110)$. Similarly, no considerable differences could be detected among the study population in terms of MACCEs (cardiacrelated death, late myocardial infarction, major hemorrhagic events, and cerebrovascular accidents) occurrence at the 24-month follow-up with $P=.167, .373, .705$ and .865 respectively. Again, there was no significant difference between the two study groups regarding readmission for cardiac causes at year one or the second year when they were compared $(P=$ .764 and .436 respectively).

\section{Follow-up Data}

At the 24-month follow-up, transthoracic echocardiography depicted a better hemodynamic performance of TAVR compared with the conventional bioprosthesis in terms of transaortic peak gradient $(\mathrm{G} 1=23.7 \pm 11.7$ versus $\mathrm{G} 2=19.5$ \pm 12.4 versus $\mathrm{G} 3=15.3 \pm 7.5 \mathrm{mmHg} ; P=.01$ ), whereas the mean gradient was similar among the groups $(\mathrm{G} 1=11.4 \pm 6$ versus $\mathrm{G} 2=10.8 \pm 6.8$ versus $\mathrm{G} 3=8.6 \pm 4.2 \mathrm{mmHg} ; P=.07$ ) (Table 4). Furthermore, a degree of at least moderate periprosthetic aortic regurgitation was found mainly in patients undergoing TAVR implantation (5 patients [9\%]), whereas such a complication did not occur in the group receiving a conventional bio or mechanical prosthesis $(P=.028)$. At the 24-month follow-up, Kaplan-Meier survival analysis comparing groups with the log-rank test showed no differences between the two groups with $P=.121$ ) (Figure).

\section{DISCUSSION}

Surgical aortic valve replacement can be done in high-risk patients. Its efficacy and safety is already proven and one can expect very good outcomes post SAVR evidenced by hospital mortality rate, ranging between 0 and $8 \%$ and a 1 -year survival rate of $-90 \%$ of patients in most of the recently reported series [Grossi 2008; Dewey 2008; Kapadia 2009; Thourani 2011].

On the other hand, an emerging option in the last few years of transcatheter approach for aortic valve replacement in high-risk and/or inoperable patients has been widely reported in the literature and has been accepted as class one indication for TAVI in the most recent guidelines [Nishimura 2017]. Its 30 - day mortality rate ranges between $6 \%$ and $12 \%$ and a 1-year survival rate around $80 \%$ in some registries Smith 2011; Rodés-Cabau 2012; Cao 2013]. Owing to the excellent and favorable results of this new approach, the therapeutic armamentarium for high-risk surgical or inoperable patients should include TAVR as evidenced by consensus statements on valvular heart disease [Vahanian 2012; Holmes Jr 2012].

The use of a transcatheter approach in patients other than those at high surgical risk or those who are deemed inoperable is therefore hotly debated [D'Onofrio 2012, Piazza 2013, Latib 2012]. Thus, our study was constructed to investigate and analyze both the early and mid-term (2-year follow-up) clinical outcomes of TAVR as an emerging option versus conventional SAVR in a population with an intermediate- to 
Table 5. MACCE and Readmission at Midterm Follow-up

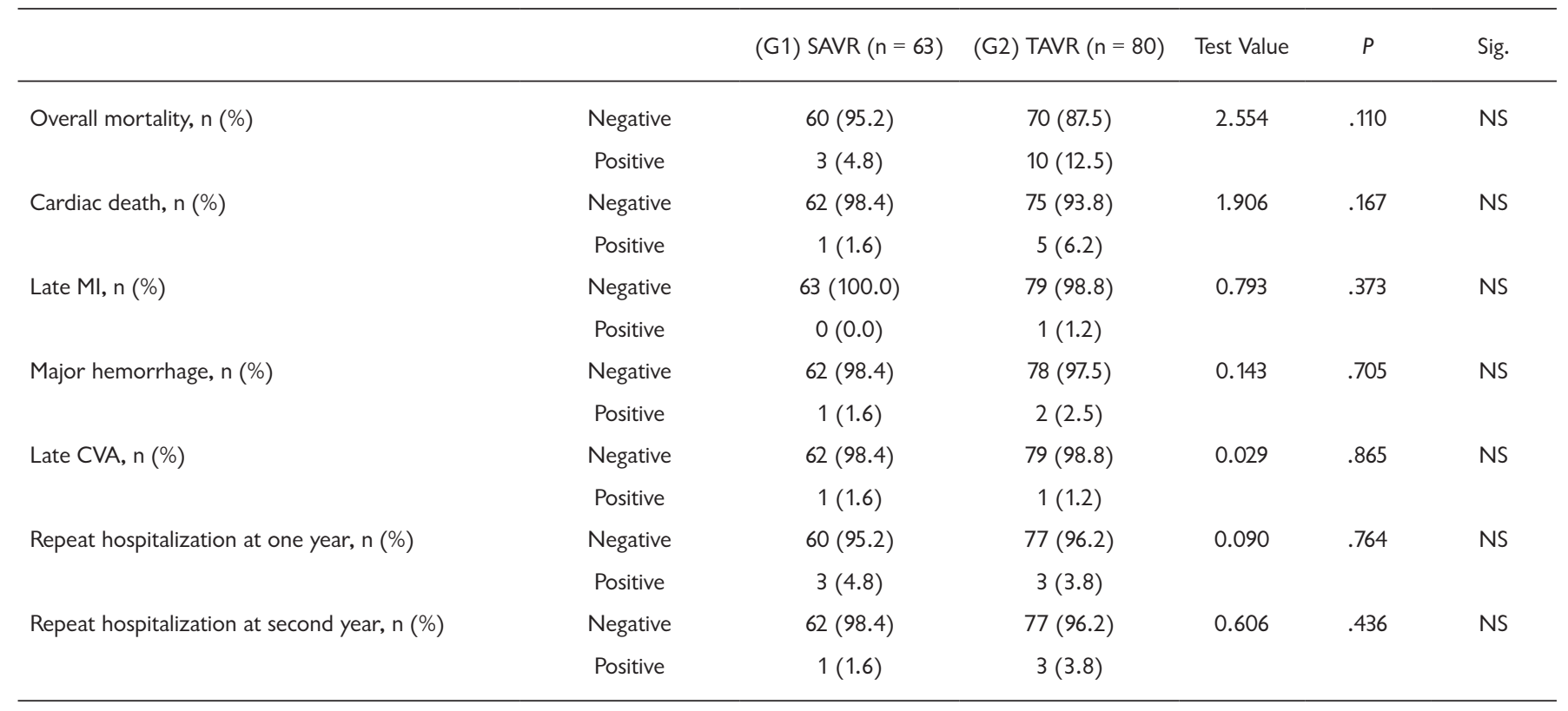

MACCEs indicates major adverse cardiac and cerebrovascular events; MI, myocardial infarction; CVA, cerebrovascular accident; NS, not significant; S, significant; HS, highly significant.

high-risk profile. Multidisclipinary evaluation, included the assessment of patients' fitness for surgery, anatomy, and degree of atherosclerotic disease of the aorta and peripheral vessels, as previously outlined in the Methods section, was the strategy followed. Even if this study is retrospective and done on a relatively small sample size, it offers some important insights into this current and controversial issue.

Most of the patients' baseline characteristics were similar among the two groups, in terms of sex, left ventricular ejection fraction (LVEF), body mass index (BMI), peripheral vascular disease, diabetes, atrial fibrillation, renal impairment, syncope, and hemodynamic parameters. However, because of the non-randomized nature of our study, some variables were significantly different between the two cohorts; in particular, patients undergoing TAVR were more likely to have COPD; meanwhile, those undergoing surgery had a higher incidence of pulmonary hypertension.

As expected, among the postoperative complications, we found a very high incidence of need for permanent pacemaker implantation in $\sim 25 \%$ of patients undergoing TAVR. Similarly, vascular complications occurred solely in TAVR group compared to SAVR group who did not undergo any peripheral vascular cannulation trail, as all cases of redo SAVR were excluded from our study to eliminate the impact of redo operations on the clinical outcome of the patients. Moreover, periprosthetic aortic regurgitation occurred more frequently in TAVR group than conventional SAVR group. However, hospital mortality was acceptably low and comparable between the two cohorts.

An explanation of the significantly high incidence of conductive tissue injury in TAVR patients is blind lateral displacement of aortic annulus calcifications that occurs during TAVR (during both balloon aortic valvuloplasty and valve deployment), rather than their physical removal or decalcification process as usually performed in SAVR, which could explain the greater incidence of this injury. Bear in mind that self-expandable devices were associated with a significantly greater incidence of pacemaker implantation than balloon-expandable valves. This is a crucial advantage of open heart surgery and SAVR technique [Ledwoch 2013]

Now, to analyze the statistically significant difference of periprosthetic aortic regurgitation seen after AVR, which was considerably lower in the SAVR patients compared with that in the TAVR patients. It is a very controversial issue, notably after the results of the PARTNER trial were published, showing at 2 years that even a mild degree of AR significantly worsens patient survival [Kodali 2012].

Colli and colleagues found that the presence and distribution of calcium within the aortic annulus has been demonstrated to strongly predict AR after TAVR. Therefore, SAVR is advantageous where the surgeon is able to physically remove or decalcify the aortic annulus entirely, decreasing the possibility of paravalvular leak to the minimum [Colli 2011]. We also agree that this issue must be solved before TAVR is indicated to include a lower-risk category of patients.

Contrary to D'Onofrio et al in 2013, we did not find any significant difference in our hospital mortality between SAVR and TAVR when compared. He explained his finding, also different from that of the PARTNER trial 3 by that his patient characteristics were different from those in the PARTNER trial; in particular, the logistic Euroscore of the PARTNER SAVR patients was $29 \%$, but in his study, it was 


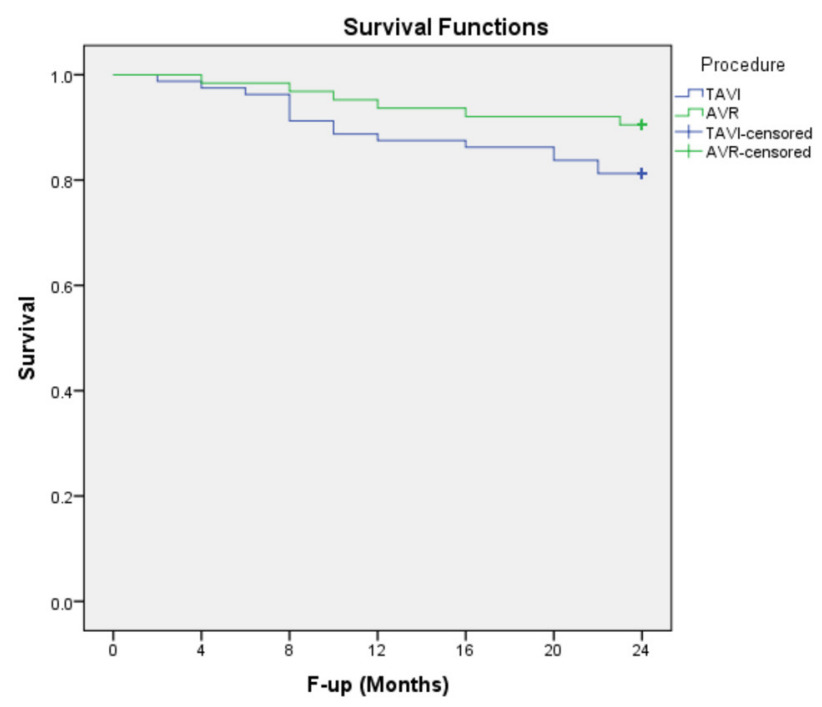

Overall survival.

$18 \%$, and this could explain the different hospital mortality rate between these 2 studies [D'Onofrio A 2013]. In our study, the EUROscore was higher in TAVR $(\mathrm{G} 2=23.33 \pm$ 8.97 versus $\mathrm{G} 1=17.83 \pm 7.09, P=.000)$ and the hospital mortality was comparable (G1 $=0 \%$ versus $\mathrm{G} 2=4.5 \%, P=.072)$. However, the absolute absence of hospital mortality in SAVR group and the presence of four mortalities in TAVR group may act as an alarming sign for a tendency to increase TAVR hospital mortality if the numbers were higher.

Another important finding similar to Muneretto et al [Muneretto 2015] is our finding of a lower peak gradient of TAVR population at 2-year follow up transthoracic echocardiography, which reflects better hemodynamic performance compared to SAVR. The clinical implications of such a finding should be scrutinized by larger prospective studies or trails with longer follow-up periods to prove or disprove its significance, bearing in mind that mean gradient was comparable between the two groups at the same echocardiographic examination.

At the 24-month follow-up, the overall survival as well as the survival free from MACCEs, was comparable between the two cohorts, signifying no perceived survival advantage of either strategy utilized. Therefore, there is no superiority of one over the other.

We therefore believe that surgical treatment of aortic valve stenosis by conventional SAVR currently remains the therapeutical option of choice in patients with severe aortic stenosis with an intermediate- to high-risk profile; meanwhile, additional evidence is needed before the use of TAVR in this specific subset of patients could be widely accepted in clinical practice.

Any center that is able to offer their patients the two therapeutic options can select the most appropriate technique, tailoring the choice to each patient and considering all crucial characteristics such as age, comorbidities, frailty, fitness to undergo surgery, and anatomy. A particularly careful evaluation is needed for patients who can benefit from either technique. An experienced heart team is therefore essential to be able to make the most appropriate choice [D'Onofrio 2013].

\section{Study Limitations}

The limitations of the present study were mainly related to the retrospective nature, and the small sample size and non-randomized nature of the current study. Longer periods of follow-up and larger patients number are needed to appreciate the effect of either strategy on survival and rate of reintervention.

\section{Conclusion}

In this study, we could not detect an advantage in survival when SAVR or TAVR were utilized in intermediate to high surgical risk patients needing aortic valve replacement for severe aortic stenosis. Further studies may be helpful in elucidating the specific subset of patients in which TAVR would be more appropriate to offer a consistent survival advantage compared with conventional AVR.

\section{REFERENCES}

Ben-Dor I, Pichard AD, Gonzalez MA, et al. 2010. Correlates and causes of death in patients with severe symptomatic aortic stenosis who are not eligible to participate in a clinical trial of transcatheter aortic valve implantation. Circulation 122:S37-42.

Cao C, Ang SC, Indraratna P, et al. 2013. Systematic review and metaanalysis of transcatheter aortic valve implantation versus surgical aortic valve replacement for severe aortic stenosis. Ann Cardiothorac Surg 2:10-23.

Colli A, D'Amico R, Kempfert J, et al. 2011. Transesophageal echocardiographic scoring for transcatheter aortic valve implantation: impact of aortic cusp calcification on postoperative aortic regurgitation. J Thorac Cardiovasc Surg 142:1229-35.

Cribier A, Eltchaninoff H, Bash A, et al. 2002. Percutaneous transcatheter implantation of an aortic valve prosthesis for calcific aortic stenosis: first human case description. Circulation 106:3006-8.

Dewey TM, Brown DL, Das TS, et al. 2008. High-risk patients referred for transcatheter aortic valve implantation: management and outcomes, Ann Thorac Surg 86:1450-6.

D'Onofrio A, Messina A, Lorusso R, et al. 2012. Sutureless aortic valve replacement as an alternative treatment for patients belonging to the 'gray zone' between transcatheter aortic valve implant-ation and conventional surgery: a propensity-matched, multicenter analysis. J Thorac Cardiovasc Surg 144:1010-6.

D’Onofrio A, Messina A, Lorusso R, et al. 2013. Conventional surgery, sutureless valves, and transapical aortic valve replacement: What is the best option for patients with aortic valve stenosis? A multicenter, propensity-matched analysis. J Thorac Cardiovasc Surg 146:1065-71.

Grossi EA, Schwartz CF, Yu PJ , et al. 2008 . High-risk aortic valve replacement: are the outcomes as bad as predicted? Ann Thorac Surg 85:102-6.

Holmes DR Jr, Mack MJ, Kaul S, et al. 2012. ACCF/AATS/SCAI/STS expert consensus document on transcatheter aortic valve replacement. J Am Coll Cardiol 59:1200-54. 
Kapadia SR, Goel SS, Svensson L, et al. 2009. Characterization and outcome of patients with severe symptomatic aortic stenosis referred for percutaneous aortic valve replacement. J Thorac Cardiovasc Surg 137:1430-5.

Kappetein AP, Head SJ, Généreux P, et al. 2012. Updated standardized endpoint definitions for transcatheter aortic valve implantation: the Valve Academic Research Consortium-2 consensus document, Eur Heart J 33:2403-18.

Kodali SK, Williams MR, Smith CR, et al. 2012. Two-year outcomes after transcatheter or surgical aortic-valve replacement. N Engl J Med 366:1686-95.

Lang RM, Bierig M, Devereux RB, et al. 2006. Recommendations for chamber quantification. Eur J Echocardiogr. 7:79-108.

Latib A, Maisano F, Bertoldi L, et al. 2012. Transcatheter vs surgical aortic valve replacement in intermediate- surgical-risk patients with aortic stenosis: a propensity score-matched case-control study. Am Heart J 164:910-7.

Ledwoch J, Franke J, Gerckens U, et al. 2013. Incidence and predictors of permanent pacemaker implantation following transcatheter aortic valve replacement: analysis from the German Transcatheter Aortic Valve Interventions Registry. Catheter Cardiovasc Interv, Wiley online library 82:E569-77.

Leon MB, Smith CR, Mack M, et al. 2010. Transcatheter aortic-valve implantation for aortic stenosis in patients who cannot undergo surgery. N Engl J Med 363:1597-607.

Muneretto C, Bisleri G, Moggi A, et al. 2015. Treating the patients in the 'grey-zone' with aortic valve disease: a comparison among conventional surgery, sutureless valves and transcatheter aortic valve replacement. J Thorac Cardiovasc Surg 20: 90-5.

Nishimura RA, Otto CM, Bonow RO, et al. 2017. AHA/ACC Focused Update of the 2014 AHA/ACC Guideline for the Management of Patients with Valvular Heart Disease: A Report of the American College of Cardiology/American Heart Association Task Force on Clinical Practice Guidelines. Circulation 136:12.
Piazza N, Kalesan B, van Mieghem N, et al. 2013. A 3-center comparison of 1-year mortality outcomes between transcatheter aortic valve implantation and surgical aortic valve replacement on the basis of propensity score matching among intermediate-risk surgical patients. JACC Cardiovasc Interv 6:443-51.

Rodés-Cabau J, Urena M, Nombela-Franco L. 2012. Indications for transcatheter aortic valve replacement based on the PARTNER trial. Rev Esp Cardiol (Engl Ed) 65:208-14.

Smith CR, Leon MB, Mack MJ, et al. 2011. PARTNER Trial Investigators.transcatheter versus surgical aortic valve replacement in high-risk patients. N Engl J Med 364:2187-98.

Thourani VH, Ailawadi G, Szeto WY, et al. 2011. Outcomes of surgical aortic valve replacement in high-risk patients: a multiinstitutional study. Ann Thorac Surg 91:49-55.

Turina J, Hess O, Sepulcri F, et al. 1987. Spontaneous course of aortic valve disease. Eur Heart J 8:471-83.

Vahanian A, Al fieri O, Andreotti F, et al. 2012. Guidelines on the management of valvular heart disease (version 2012): the Joint Task Force on the Management of Valvular Heart Disease of the European Society of Cardiology (ESC) and the European Association for Cardio-Thoracic Surgery (EACTS). Eur J Cardiothorac Surg 42:S1-44.

Zoghbi WA, Chambers JB, Dumesnil JG et al. 2009. Recommendations for evaluation of prosthetic valves with echocardiography and Doppler ultrasound: a report from the American Society of Echocardiography's Guidelines and Standards Committee and the Task Force on Prosthetic Valves, developed in conjunction with the American College of Cardiology Cardiovascular Imaging Committee, Cardiac Imaging Committee of the American Heart Association, the European Association of Echocardiography, a registered branch of the European Society of Cardiology, the Japanese Society of Echocardiography and the Canadian Society of Echocardiography, endorsed by the American College of Cardiology Foundation, American Heart Association, European Association of Echocardiography, a registered branch of the European Society of Cardiology, the Japanese Society of Echocardiography, and Canadian Society of Echocardiography. J Am Soc Echocardiogr. 22:975-1014. 